\title{
Hypomethylated Rab27b is a progression-associated prognostic biomarker of glioma regulating MMP-9 to promote invasion
}

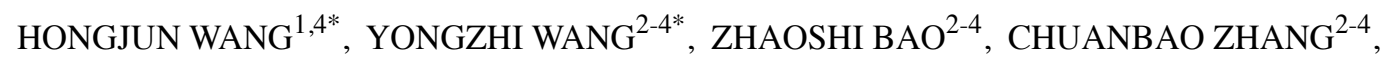 \\ YANWEI LIU ${ }^{2-4}$, JINQUAN CAI ${ }^{1,4}$ and CHUANLU JIANG ${ }^{1,4}$ \\ ${ }^{1}$ Department of Neurosurgery, The Second Affiliated Hospital of Harbin Medical University, Harbin, Heilongjiang; \\ ${ }^{2}$ Department of Neurosurgery, Beijing Tiantan Hospital, Capital Medical University, Beijing; \\ ${ }^{3}$ Beijing Neurosurgical Institute, Beijing; ${ }^{4}$ Chinese Glioma Cooperative Group (CGCG), Beijing, P.R. China
}

Received April 6, 2015; Accepted June 23, 2015

DOI: $10.3892 /$ or.2015.4125

\begin{abstract}
In the present study, in order to analyze abnormalities in DNA methylation in glioma, we utilized a large cohort methylation microarray (119 glioma samples). Genes associated with tumor grade progression were screened through Significance Analysis of Microarrays (SAM) in the methylation microarray. We found that Rab27b was hypomethylated in high-grade glioma (anaplastic gliomas and glioblastomas) compared with low-grade glioma (astrocytoma, oligodendrocytoma and oligoastrocytoma) ( $\mathrm{p}=0.02)$. In 52 glioma samples, we determined both the methylation status of the Rab27b promoter region and protein expression, and confirmed a negative correlation between the methylation status and expression $(\mathrm{p}<0.01)$. Immunohistochemistry of 91 gliomas revealed that the Rab27b expression scores of high-grade glioma were higher than scores of low-grade gliomas $(\mathrm{p}<0.01)$. In high-grade gliomas, patients haboring Rab27b hypomethylation or overexpression had unfavorable survival prognosis. Transwell invasion assays identified that invasive cell number of glioma U87 and LN229 cells decreased when Rab27b was knocked down. Decreased invasion partly resulted from reduced expression and activation of MMP-9 after Rab27b knockdown. Downregulation of Rab27b also suppressed tumor growth in vivo. Hypomethylated Rab27b was identified as a progression-associated and prognostic molecular marker of glioma.
\end{abstract}

Correspondence to: Dr Chuanlu Jiang, Department of Neurosurgery, The Second Affiliated Hospital of Harbin Medical University, 246 Xuefu Road, Harbin, Heilongjiang 150086, P.R. China E-mail: jc16688jcl@163.com

*Contributed equally

Key words: glioma, Rab27b, hypomethylation, prognosis

\section{Introduction}

Despite the application of surgery and chemotherapy and/or radiotherapy, the prognosis of patients with malignant glioma is still extremely poor. Glioblastoma multiforme (GBM) is the most malignant glioma, the median survival of which is approximately 1 year (1-3). The invasive growth pattern of glioma cells is partially responsible for its poor response to treatment (4). Some characteristic oncogenes may take responsibility for the infiltration and invasion.

Epigenetic mechanisms are important factors in the regulation of gene expression in tumors. To date, research has discovered many epigenetic mechanisms, for example promoter hypermethylation/hypomethylation, histone modifications, or non-coding RNA expression (5). Moreover, DNA methylation is typically a more stable and inheritable epigenetic pattern, and is an important hallmark of tumor development and progression (6-8). Although the important role of hypermethylation in the silencing of tumor-suppressor genes is now well-documented (9), a decrease in the level of methylation also contributes to numerous types of human cancer $(10,11)$.

The RAS superfamily of small GTPases includes 5 groups: Ras, Rho, Rab, Ran and Arf. Rab family proteins are important in regulating signal transduction and cellular processes, such as differentiation, proliferation, vesicle transport, nuclear assembly and cytoskeleton formation. In addition, various Rab proteins are important for the adhesion and migration of cancer cells. Rab27b can control vesicle exocytosis and release important growth regulators into the tumor microenvironment, regulating invasive growth and metastasis in human breast tumors (12). Yet, the role of Rab27b in glioma remains unclear.

In the present study, the promoter methylation status of Rab27b was evaluated in our methylation microarray (119 samples) and 52 independent samples. Correlation of Rab27b methylation with clinical data was assessed. Rab27b expression was also detected to determine whether promoter hypomethylation was correlated with the high expression level in gliomas. Furthermore, we explore the probable mechanistic link through experiments in vitro and in vivo. 


\section{Materials and methods}

Ethics. Written informed consent from each donor or the next of kin was obtained. This study was performed following approval of the Ethics Committee of the Beijing Tiantan Hospital and is in compliance with the Helsinki Declaration.

Datasets used in this study. All patients were from the Chinese Glioma Genome Atlas (CGGA). All of the patients selected in the present study underwent surgical resection between January 2006 and December 2010 and subsequently received concomitant and adjuvant temozolomide and radiotherapy. Data concerning overall survival (OS), defined as the period from operation to death, were collected mainly when patients visited the clinics or by phone interview with patients and/or their relatives. Patients who died of non-primary diseases were excluded (13). Tumor tissue samples were obtained by surgical resection before treatment with radiation and chemotherapy. Respected specimens were snap-frozen and stored in liquid nitrogen until nucleic acid extraction. Only samples with $>80 \%$ tumor cells were selected. The microarray data set was deposited in the Gene Expression Omnibus (GEO) (accession no. GSE53227 and GSE53228) according to 'minimum information about a microarray experiment' (MIAME) guidelines.

DNA extraction. All of the tissue samples were immediately snap-frozen in liquid nitrogen after surgery. A hematoxylin and eosin-stained frozen section was prepared for assessment of the percentage of tumor cells before DNA extraction. Genomic DNA was isolated from frozen tumor tissues using the QIAamp DNA Mini kit (Qiagen) according to the manufacturer's instructions. DNA concentration and quality were measured using the NanoDrop ND-1000 spectrophotometer (NanoDrop Technologies, Houston, TX, USA) (8).

Genome-wide DNA methylation profiling. A series of 119 glioma samples (63 low-grade gliomas, 33 anaplastic gliomas and 23 glioblastomas) were assessed by methylation microarray. We used the Illumina Infinium Human Methylation 27 BeadChips (Illumina Inc.) as previously described (14). The BeadChip contains 27,578 highly informative CpG sites covering >14,000 human RefSeq genes. This allows researchers to interrogate all of these sites per sample at a single nucleotide resolution. Bisulfite modification of DNA, chip processing and data analysis were performed following the manufacturer's manual at the Wellcome Trust Centre for Human Genetics Genomics Laboratory (Oxford, UK). The array results were analyzed with the BeadStudio software (Illumina Inc.).

Bisulfite sequencing PCR (BSP). Fifty-six glioma samples (4 normal brain tissues and 20 low-grade and 32 high-grade gliomas) were measured by BSP and immunohistochemistry (IHC). Purified DNA was treated with sodium bisulfite (Sigma, Phoenix, AZ, USA). The PCR products were confirmed by agarose gel electrophoresis and visualized using ethidium bromide staining. The primers used were: 5'-AGTAAGATTT GTTTGAGGTGAGTTT-3' and 3'-AAAACAACTACTTATC TCCTCAACC-5'. There were 11 CPG sites in the amplified region (18q21.2, 54715778-54715993). The specific experi- mental steps were in accordance with the test method from the EZ DNA Methylation-Direct kit (Zymo Research).

Immunohistochemistry. Ninety-one glioma samples included 30 low-grade and 20 anaplastic gliomas and 41 glioblastomas. IHC was performed as previously described (17). The degree of immunostaining of the sections was viewed and scored separately by two independent investigators. The scores were determined by combining the proportion of positively stained tumor cells and the intensity of staining. Binary decision criterion of Rab27b expression was performed as previously described $(8,16)$.

Cell lines and culture. Human glioblastoma cell lines U87 and LN229 were obtained from the Institute of Biochemistry and Cell Biology, Chinese Academy of Science (Shanghai, China). The cells were maintained in Dulbecco's modified Eagle's medium (DMEM; Gibco, Carlsbad, CA, USA) supplemented with $10 \%$ fetal bovine serum (FBS) and incubated at $37^{\circ} \mathrm{C}$ in $5 \% \mathrm{CO}_{2}$. Upon $80 \%$ confluency, the cells were starved in DMEM with $1 \%$ FBS for $24 \mathrm{~h}$ and maintained in this low serum condition for the course of treatment (8).

Rab27b gene knockdown by siRNA. Specific oligos targeting the Rab27b gene were selected (OriGene Technologies, Inc.). siRNA1, the most efficient one, screened from 3 siRNAs, was used to knockdown Rab27b (5'-AAA GGT GTG GTT TAT AAT GCA-3'), and a RNA interference negative control was purchased from Qiagen (Venlo, The Netherlands). Logarithmically growing cells were seeded at a density of $10^{5}$ cells $/ 6-\mathrm{cm}$ dish and transfected with $5 \mu \mathrm{mol} \mathrm{Rab} 27 \mathrm{~b}$ siRNA using Lipofectamine 2000 (Invitrogen) according to the manufacturer's instructions. Forty-eight hours later, the cells were used for in vitro functional assays as described below.

Transwell invasion assay. The Transwell invasion assay was conducted in 24-well cell culture chambers using Transwell inserts (Corning Life Sciences, Corning, NY, USA; BD Biosciences, San Jose, CA, USA) with an $8-\mu \mathrm{m}$ pore membrane precoated with Matrigel (BD Biosciences). U87 and LN229 cells were plated at the density of $1 \times 10^{4} /$ upper well in $200 \mu \mathrm{l}$ culture medium (DMEM, no FBS), as well as control group and siRNA group, respectively. The lower chamber was filled with $500 \mu 1$ medium (DMEM, 12\% FBS). The cells were allowed to invade for $24 \mathrm{~h}$, after which, the non-invading cells with Matrigel matrix were removed from the upper surface of the membrane by scrubbing with a cotton-tipped swab. Cells on the lower surface of the filter were fixed for $30 \mathrm{~min}$ in methanol and glacial acetic acid mixture (3:1), air-dried briefly, and stained with crystal violet. The mean number of invaded cells was counted from five preselected microscopic fields at x200 magnification; all experiments were performed in triplicate.

Western blot analysis. After cell treatment, cell lysates were prepared via lysis buffer, electrophoresed onto SDS-polyacrylamide gels, and transferred to polyvinylidene difluoride membranes (4). Membranes were probed with antibodies against Rab27b (Proteintech) and 
A

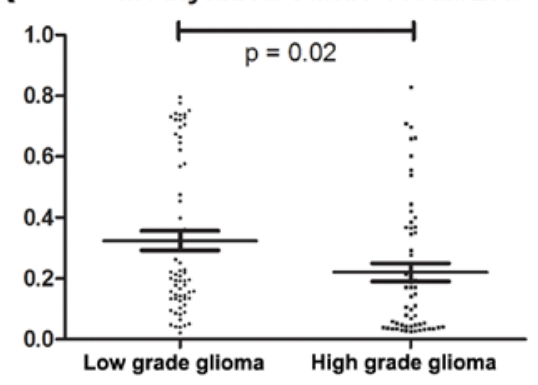

Overall survival

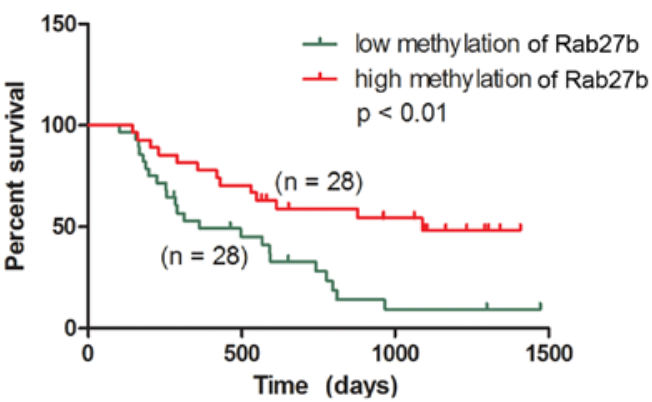

C
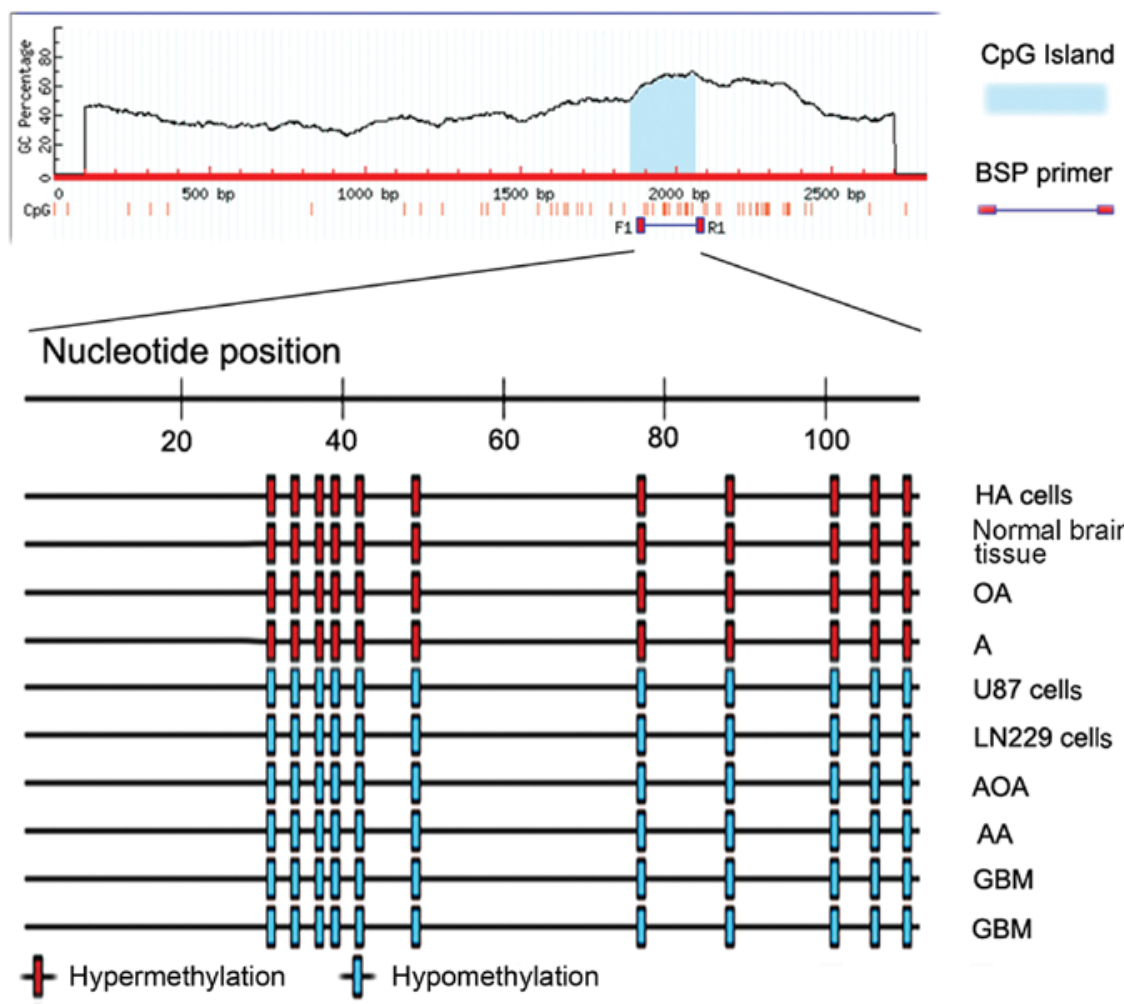

HA cells

Normal brain

tissue

$\mathrm{OA}$

A

U 87 cells

LN229 cells

AOA

AA

GBM

GBM

Figure 1. Rab27b hypomethylation is associated with tumor grade progression and poor prognosis as determined in the CGGA data. (A) The methylation level of the Rab27b promoter was significantly lower in high-grade glioma than that in low-grade gliomas. (B) In high-grade gliomas, patients with a low methylation level of Rab27b had shorter OS than those with high methylation. (C) There was a big CpG island located in upstream of the Rab27b promoter region. Methylation analysis using BSP method showed differential methylation levels in different cell lines and glioma tissues. CGGA, Chinese Glioma Genome Atlas; OS, overall survival; BSP, bisulfite sequencing PCR. Note: Hypermethylation, HA cells (normal brain cells), normal brain tissue, low-grade glioma (OA, oligoastrocytoma; A, astrocytoma); Hypomethylation, glioma cell lines (U87 and LN229); high-grade glioma (AOA, anaplastic oligoastrocytoma; AA, anaplastic astrocytoma; GBM, glioblastoma multiforme).

glyceraldehyde-3-phosphate dehydrogenase (GAPDH, A-3; Santa Cruz Biotechnology) at dilutions of 1:1,000. Blots were detected with horseradish peroxidase-labeled anti-rabbit antibodies (1:5,000 dilution) developed using enhanced chemiluminescence (ECL) reagents (Amersham Pharmacia, UK). The primary antibody against MMP-9 (Oncogene, Boston, MA, USA), was used to examine protein expression.

Determination of gelatinase activity. Cells ( $1 \times 10^{6} / 35-\mathrm{mm}$ dish) undergoing Rab27b siRNA treatments or the negative control were subsequently incubated in $2 \mathrm{ml}$ serum-free medium for $24 \mathrm{~h}$. The supernatants were centrifuged at $10,000 \mathrm{rpm}$ for $5 \mathrm{~min}$ to remove cell debris and their protein contents were determined using Bradford reagent (Bio-Rad, USA).
Gelatinolytic activity of MMP-9 and MMP-2 was examined by gelatin zymography. Briefly, equal amounts $(10 \mu \mathrm{g})$ of protein were mixed with SDS gel-loading buffer and then loaded without reduction or heating onto $10 \%$ polyacrylamide gels containing $0.1 \%$ gelatin (Sigma). To detect specific activity, SDS-PAGE was carried out. After running, the gels were renatured for $1 \mathrm{~h}$ in $0.25 \%$ Triton X-100 at room temperature with gentle agitation, and then incubated in developing buffer (50 mM Tris- $\mathrm{HCl}, 100 \mathrm{mM} \mathrm{NaCl}, 10 \mathrm{mM} \mathrm{CaCl}_{2}$ and $0.02 \%$ $\mathrm{NaN}_{3}, \mathrm{pH} 7.5$ ) for $24 \mathrm{~h}$ at $37^{\circ} \mathrm{C}$. After briefly washing in water, the gels were stained with Coomassie blue R-250 for $1 \mathrm{~h}$ to reveal the gelatinolytic activity. Gels were destained with $40 \%$ methanol and 5\% acetic acid until clear white bands against a blue background were visible. 
Table I. Methylation status of Rab27b was negatively correlated with grade and Rab27b expression.

\begin{tabular}{lcc}
\hline & \multicolumn{2}{c}{$\begin{array}{c}\text { Methylation status of } \\
52 \text { glioma samples }\end{array}$} \\
\cline { 2 - 3 } Characteristics & $\begin{array}{c}\text { Low } \\
\text { n/total (\%) }\end{array}$ & $\begin{array}{c}\text { High } \\
\text { n/total (\%) }\end{array}$ \\
\hline Low grade & $6 / 20(30)$ & $14 / 20(70)$ \\
High grade & 22/32 $(68.8)$ & $10 / 32(31.2)$ \\
& \multicolumn{2}{c}{ Methylation status of } \\
& \multicolumn{2}{c}{32 high-grade samples } \\
\cline { 2 - 3 } Characteristics & Low & High \\
\hline Rab27b low expression & n/total (\%) & n/total (\%) \\
Rab27b high expression & 8/22(22.7) & $17 / 22(77.3)$ \\
\hline
\end{tabular}

${ }^{\mathrm{a}} \mathrm{p}<0.01 ;{ }^{\mathrm{b}} \mathrm{p}<0.01$.

Nude mouse tumor xenograft model and treatment with siRNA of Rab27b. LN229 glioma cells were subcutaneously injected into 5-week-old female nude mice (Cancer Institute of the Chinese Academy of Medical Science). When the tumor volume reached $50 \mathrm{~mm}^{3}$, the mice were randomly divided into two groups (6 mice/group). Each group was treated with siRNA of Rab27b or negative control oligo in $10 \mu \mathrm{l}$ Lipofectamine through local injection of the xenograft tumor at multiple sites. The treatment was performed once every 3 days for 21 days. The tumor volume was measured with a caliper twice a week, using the following formula: Volume = length $\mathrm{x} \mathrm{width}^{2} / 2$.

Statistical analysis. Significant Analysis of Microarrays (SAM) was used for the genes significantly differentially methylated between high- and low-grade gliomas. The t-test or Chi-square test was applied for statistical analysis of the correlation between two independent variables. Survival curves were estimated using the Kaplan-Meier method, and statistical differences were evaluated using the two-sided logrank test. A $p<0.05$ was considered significant.

\section{Results}

Rab27b methylation status is correlated with tumor grade progression and prognosis. In a microarray of 119 glioma samples, we found that Rab27b was significantly hypomethylated in high-grade gliomas compared with low-grade gliomas ( $\mathrm{p}=0.02$, Fig. 1A). The methylation status of Rab27b was correlated with OS in the high-grade glioma samples through Kaplan-Meier survival curve analysis ( $\mathrm{p}<0.01$, Fig. 1B). There was a big $\mathrm{CpG}$ island located in nearly 2,000 bp upstream of the Rab27b promoter region (Fig. 1C). These CG sites in the CpG island were detected by BSP method, and glioma cell lines (U87 and LN229) harbored a low methylation level than the level in the glio cell line (HA cells). Similar to these results, the methylation levels in these sites from the high-grade glioma tissues were lower than levels in the low-grade glioma and normal brain tissues.

The methylation status of Rab27b was significantly lower in high-grade gliomas (68.8\%) compared with the status in low-grade gliomas $(31.2 \%)$ (Table I, $\mathrm{p}<0.01)$, which was significantly associated with glioma grade progression. In 32 high-grade glioma samples, $80 \%$ showed low Rab27b promoter methylation accompanied by high Rab27b protein expression detected by the IHC method, and the remaining samples showed high Rab27b promoter methylation along with low protein expression.

Rab27b expression is associated with glioma grade progression and confers a poor prognosis to high-grade glioma patients with high Rab27b expression. We performed IHC of 91 glioma samples from mainland Han Chinese patients. We analyzed the correlation between Rab27b protein expression and the histological stage of the gliomas. Rab27b expression ranged from low to high along with the grade of progression of the gliomas (p<0.01, Fig. 2A and C). Survival analysis showed that patients with high Rab27b expression had significantly shorter OS $(p=0.01)$ than those with low expression in the high-grade glioma patients (Fig. 2B).

Rab27b promotes glioma invasion via activation of MMP-9 but not MMP-2. Western blot analysis identified that Rab27b was significantly knocked down by siRNA compared with the control group in the U87 cells (Fig. 3A). To confirm the effect of Rab27b on the regulation of intracellular molecules involved in aggressive cell behavior, we examined MMP-9 expression. MMP-9 expression was downregulated following Rab27b knockdown when compared to the expression levels in the control group. The gelatinase activity assay showed that MMP-9 activation was weakened but not MMP-2 following Rab27b knockdown (Fig. 3B). After siRNA knockdown of Rab27b in the glioma cell lines, the U87 and LN229 cell lines were used for Transwell invasion assay. siRNA significantly attenuated the effect of Rab27b on cell invasion of the U87 cells $(\mathrm{p}<0.01$, Fig. $3 \mathrm{C})$ as well as the LN229 cells $(\mathrm{p}<0.01)$, respectively.

Rab27b promotes glioma growth in vivo. To investigate the potential impact of Rab27b expression in vivo, a LN229 cell xenograft model was utilized. The Rab27b-treated group displayed a significant growth reduction, whereas tumor growth was not impacted by the negative control (Fig. 4A). Tumor mass (Fig. 4B) was significantly higher in the control tumors when compared with those treated with Rab27b. IHC analysis confirmed downregulated expression of Rab27b in the treated group (Fig. 4C). Rab27b-treated glioma model displayed decreased expression of MMP-9 compared with the control. These data indicated that Rab27b knockdown in vivo functioned similarly as the effect in vitro.

\section{Discussion}

In the present study, we investigated the methylation and expression level of Rab27b in 3 independent cohorts, including 262 glioma patients. The methylation level of the Rab27b promoter was associated with tumor grade progression and 
A

A Rab27b expression by IHC

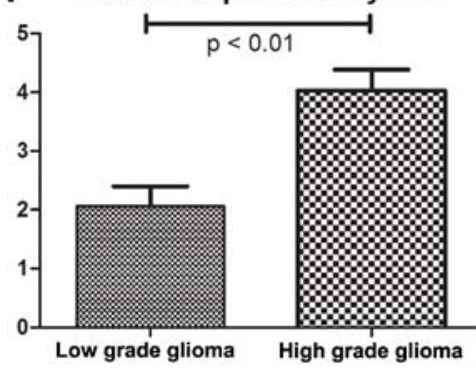

C

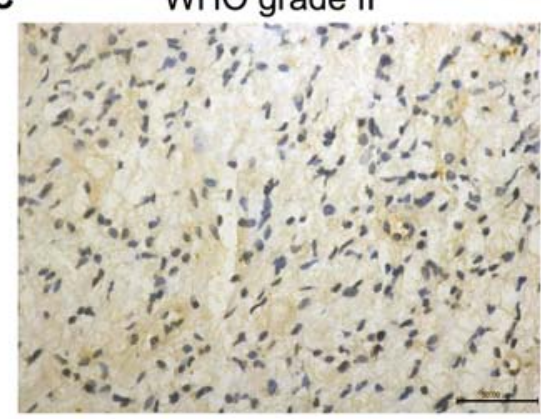

B

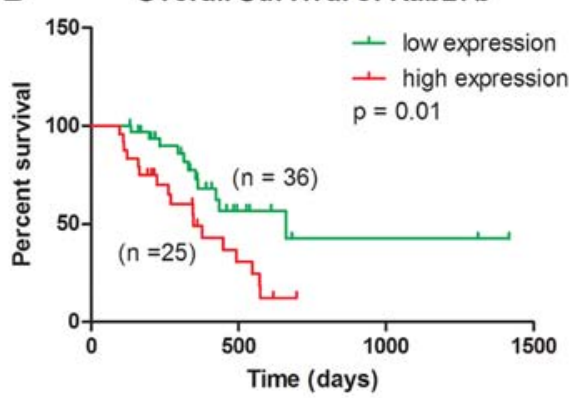

WHO grade IV

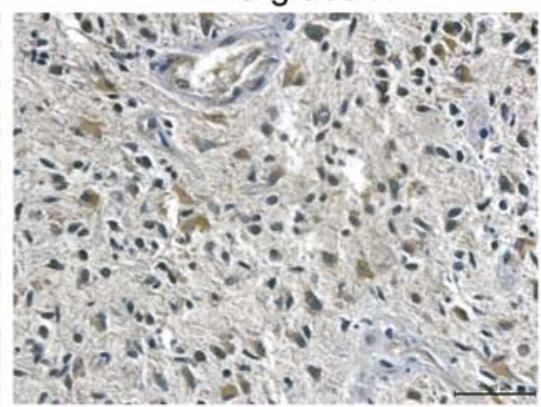

Figure 2. Rab27b expression is correlated with glioma grade progression and prognosis. (A) The expression of Rab27b was significantly higher in the high-grade glioma patients than those with low-grade glioma. (B) Patients with high Rab27b expression had significant shorter overall survival than those with low expression in the high-grade glioma cases. (C) IHC showed that high-grade gliomas exhibited higher Rab27b expression compared with low-grade gliomas. IHC, immunohistochemistry.
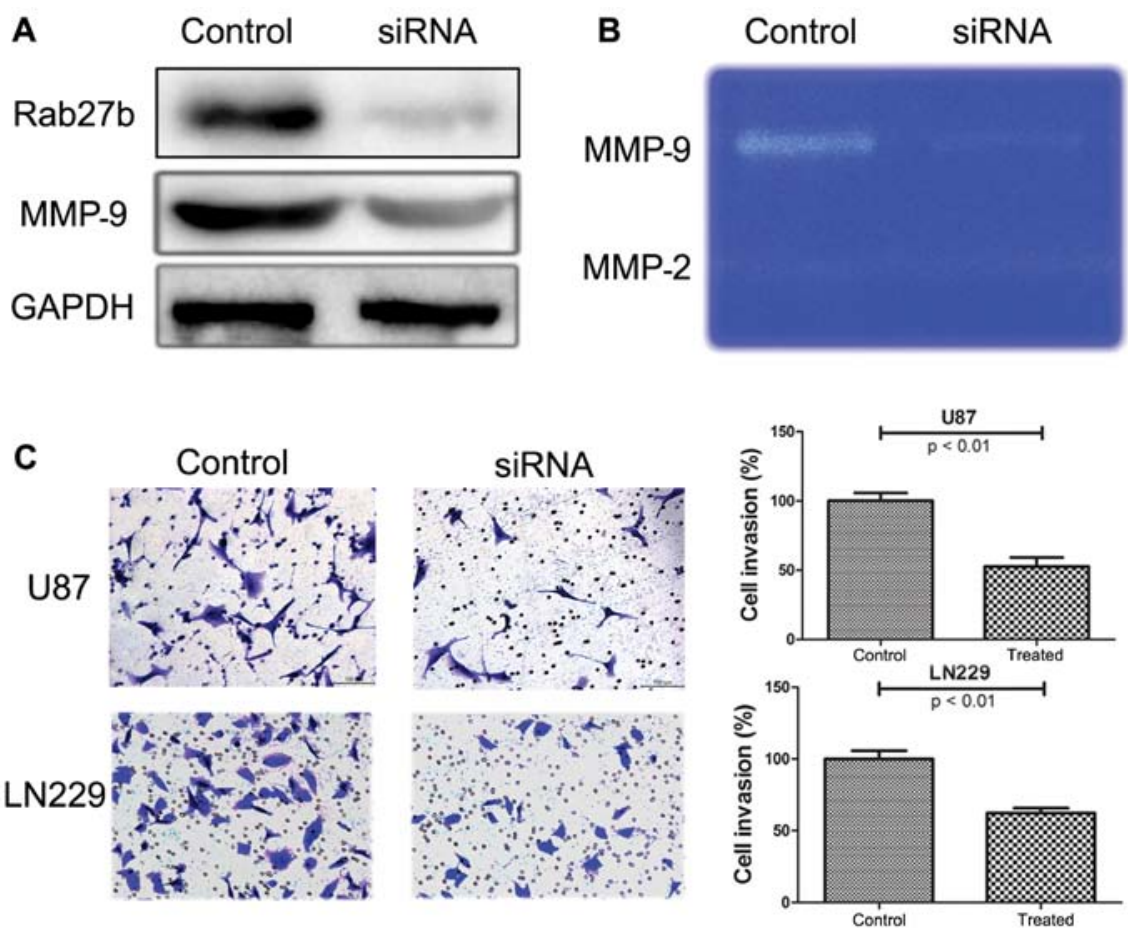

Figure 3. Rab27b promotes the invasive abilities of U87 and LN229 cells by regulating MMP-9. (A) Following knockdown of Rab27b by siRNA, western blot analysis showed that MMP-9 expression was reduced compared with the level in the control group. (B) Gelatinase activity assay showed that MMP-9 activation was weakened but not MMP-2 in the U87 cells following Rab27b knockdown. (C) Invasion of U87 and LN229 cells was assessed by Transwell invasion assay. The number of invading cells was significantly less in the siRNA group compared with the control group. The number of invading cells are shown in the histogram. $\mathrm{p}<0.01$ vs. control, respectively.

survival in malignant gliomas. We further detected the difference in expression of Rab27b in different grade gliomas, and observed a significant increase in Rab27b expression from low- to high-grade gliomas. Rab27b was an independent unfavorable prognostic factor for the patients with high-grade glioma. In vitro analysis revealed that Rab27b promoted the 

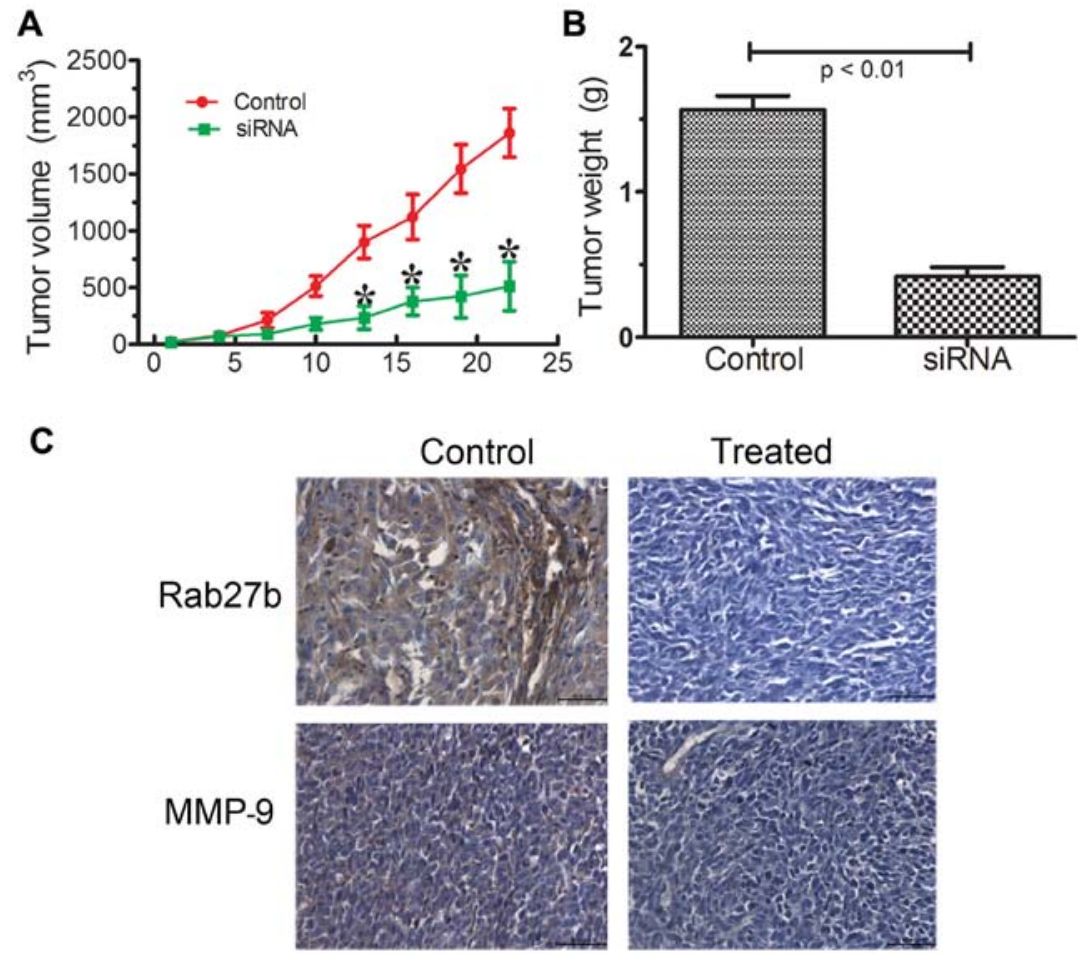

Figure 4. Rab27b promotes glioma tumor growth in vivo. (A) LN229 tumors were treated with siRNA of Rab27b and the negative control. Growth curves of the siRNA Rab27b-treated LN229 tumors $(\mathrm{n}=6)$ vs. the negative control group $(\mathrm{n}=6)$. (B) Tumors were resected and tumor mass was determined 22 days following implantation. Data indicate that the tumor mass was decreased in the siRNA-Rab27b-treated LN229 tumors compared with the negative control group. (C) IHC assay for Rab27b expression in the negative control LN229 tumors vs. the siRNA Rab27b-treated xenografts. MMP-9 expression was downregulated accordingly in the treated group. IHC, immunohistochemistry.

invasion of glioma cells, at least in part, by activating MMP-9. As far as we know, this is the first study on the methylation status, expression difference, and function of Rab27b in glioma.

A large body of evidence demonstrates that DNA methylation plays a role in gene regulation in animal cells. Hypomethylation of oncogenes and hypermethylation of suppressor gene promoters are pivotal alterations in tumor development (17). Genome-wide DNA methylation profiling has revealed a glioma-CpG island methylator phenotype $\left(\mathrm{G}-\mathrm{CIMP}^{+}\right)$subtype that has a strong positive correlation with survival prognosis in GBM patients (18), however the role of promoter demethylation remains obscure. In the present study, a genome-wide DNA methylation microarray including 119 gliomas was performed. We found that the methylation level of Rab27b in high-grade gliomas was significantly lower than that in low-grade ones. Kaplan-Meier survival curve analysis showed that patients with a low methylation level of Rab27b had shorter OS compared to the high methylation group in patients with high-grade glioma. To identify whether the expression of Rab27b was correlated with the status of methylation in glioma, we detected both the Rab27b expression and methylated level of the promoter region in 52 glioma tissues. As shown in Table I, the Rab27b expression was negatively associated with the promoter methylation status $(\mathrm{p}<0.01)$. In another independent cohort including 91 glioma samples, we found that patients with high-grade glioma had significant higher Rab27b expression than those patients with low-grade glioma. In 61 high-grade glioma patients, high Rab27b expression was associated with adverse outcomes.
The results indicated that glioma with Rab27b hypomethylation and higher protein expression may be a more biologically aggressive phenotype, and that elevated expression of Rab27b resulting in a shorter survival was at least partially due to hypomethylation of the Rab27b promoter region.

To date, no information has been provided concerning the Rab27b gene in gliomas, and its clinical effect on survival remains unknown. Ras-associated binding (Rab)-GTPases are members of the Ras family of small GTPases. Many Rab proteins are abnormally expressed in different cancer tissues. Rab25 has been shown to decrease apoptosis, as well as to increase both the proliferation and aggressiveness of ovarian and breast cancer (16). Rab5 is involved in EGFR signaling and was found to promote proliferation in lung adenocarcinoma cell lines (20). Rab27b was reported to control vesicle exocytosis and release important growth regulators into the tumor microenvironment, leading to regulation of invasive growth and metastasis in human breast tumors (12). We performed functional assays in malignant glioma U87 and LN229 cell lines and confirmed that Rab27b promoted the invasion of glioma cells. Furthermore, Rab27b regulated the expression and activation status of MMP-9 which characteristically participates in tumor invasion. Thus, these functional assays confirmed our hypothesis.

In summary, Rab27b promoter hypomethylation and expression are significantly correlated with the tumor grade progression of glioma. The methylation status of Rab27b was negatively associated with its expression. Patients harboring Rab27b hypomethylation and high expression had reduced OS in high-grade glioma. Rab27b promoted glioma invasion, 
at least in part, via regulation of MMP-9 expression and activation.

\section{Acknowledgements}

This research was supported by grants from the Health and Family Planning Commission Research Project of Heilongjiang province (2014-329), the Government Spending Postdoctoral Fellowship of Heilongjiang province (LRB14-422), the National High Technology Research and Development Program (2012AA02A508), the National Natural Science Foundation of China (nos. 81402052, 81201993, 81372700 and 81302200) and the International Science and Technology Cooperation Program (2012DFA30470).

\section{References}

1. Zhang W, Yan W, You G, Bao Z, Wang Y, Liu Y, You Y and Jiang T: Genome-wide DNA methylation profiling identifies ALDH1A3 promoter methylation as a prognostic predictor in G-CIMP- primary glioblastoma. Cancer Lett 328: 120-125, 2013.

2. Yan W, Zhang W, You G, Zhang J, Han L, Bao Z, Wang Y, Liu Y, Jiang C, Kang C, et al: Molecular classification of gliomas based on whole genome gene expression: A systematic report of 225 samples from the Chinese Glioma Cooperative Group. Neuro Oncol 14: 1432-1440, 2012.

3. Wang Y, Li S, Chen L, You G, Bao Z, Yan W, Shi Z, Chen Y, Yao K, Zhang W, et al: Glioblastoma with an oligodendroglioma component: Distinct clinical behavior, genetic alterations, and outcome. Neuro Oncol 14: 518-525, 2012.

4. Wang H, Wang Y and Jiang C: Stromal protein periostin identified as a progression associated and prognostic biomarker in glioma via inducing an invasive and proliferative phenotype. Int J Oncol 42: 1716-1724, 2013.

5. Jones PA and Baylin SB: The epigenomics of cancer. Cell 128: 683-692, 2007

6. Gaspar-Maia A, Alajem A, Meshorer E and Ramalho-Santos M: Open chromatin in pluripotency and reprogramming. Nat Rev Mol Cell Biol 12: 36-47, 2011.

7. Feinberg AP and Tycko B: The history of cancer epigenetics. Nat Rev Cancer 4: 143-153, 2004.

8. Wang H, Feng Y, Bao Z, Jiang C, Yan W, Wang Y, Zhang C, Liu Y, Zhang Q, Zhang W, et al: Epigenetic silencing of KAZALD1 confers a better prognosis and is associated with malignant transformation/progression in glioma. Oncol Rep 30 2089-2096, 2013.
9. Baylin SB: DNA methylation and gene silencing in cancer. Nat Clin Pract Oncol 2 (Suppl 1): S4-S11, 2005.

10. Ehrlich M: DNA methylation in cancer: Too much, but also too little. Oncogene 21: 5400-5413, 2002.

11. Cadieux B, Ching TT, Van den Berg SR and Costello JF: Genome-wide hypomethylation in human glioblastomas associated with specific copy number alteration, methylenetetrahydrofolate reductase allele status, and increased proliferation. Cancer Res 66: 8469-8476, 2006.

12. Hendrix A, Maynard D, Pauwels P, Braems G, Denys H, Van den Broecke R, Lambert J, Van Belle S, Cocquyt V, Gespach C, et al: Effect of the secretory small GTPase Rab27B on breast cancer growth, invasion, and metastasis. J Natl Cancer Inst 102: 866-880, 2010.

13. Li S, Yan C, Huang L, Qiu X, Wang Z and Jiang T: Molecular prognostic factors of anaplastic oligodendroglial tumors and its relationship: A single institutional review of 77 patients from China. Neuro Oncol 14: 109-116, 2012.

14. Hill VK, Ricketts C, Bieche I, Vacher S, Gentle D, Lewis C, Maher ER and Latif F: Genome-wide DNA methylation profiling of $\mathrm{CpG}$ islands in breast cancer identifies novel genes associated with tumorigenicity. Cancer Res 71: 2988-2999, 2011.

15. Zhang W, Qiu XG, Chen BS, Li SW, Cui Y, Ren H and Jiang T: Antiangiogenic therapy with bevacizumab in recurrent malignant gliomas: Analysis of the response and core pathway aberrations. Chin Med J (Engl) 122: 1250-1254, 2009.

16. Wang F, Yang C, Song Y, Jiang Y and Ding Z: Periostin gene polymorphisms, protein levels and risk of incident coronary artery disease. Mol Biol Rep 39: 359-367, 2012.

17. Iacob $\mathrm{G}$ and Dinca EB: Current data and strategy in glioblastoma multiforme. J Med Life 2: 386-393, 2009.

18. Laffaire J,Everhard S,Idbaih A, Crinière E, Marie Y, de Reyniès A, Schiappa R, Mokhtari K, Hoang-Xuan K, Sanson M, et al: Methylation profiling identifies 2 groups of gliomas according to their tumorigenesis. Neuro Oncol 13: 84-98, 2011.

19. Lebrand C, Corti M, Goodson H, Cosson P, Cavalli V, Mayran N, Fauré J and Gruenberg J: Late endosome motility depends on lipids via the small GTPase Rab7. EMBO J 21: 1289-1300, 2002.

20. Tomshine JC, Severson SR, Wigle DA, Sun Z, Beleford DA, Shridhar $\mathrm{V}$ and Horazdovsky BF: Cell proliferation and epidermal growth factor signaling in non-small cell lung adenocarcinoma cell lines are dependent on Rin1. J Biol Chem 284: 26331-26339, 2009. 